\title{
Vortex solid phase with frozen undulations in superconducting Josephson-junction arrays in external magnetic fields
}

\author{
Hajime Yoshino ${ }^{1}$, Tomoaki Nogawa ${ }^{2}$, Bongsoo Kim ${ }^{3}$ \\ ${ }^{1}$ Department of Earth and Space Science, Faculty of Science, Osaka University, Toyonaka 560-0043, Japan \\ ${ }^{2}$ Department of Applied Physics. School of Engineering, \\ The University of Tokyo, 7-3-1 Hongo, Bunkyo-ku, Tokyo 113-8656, Japan \\ ${ }^{3}$ Department of Physics, Changwon National University, Changwon 641-773, Korea.
}

(Dated: August 9, 2018)

\begin{abstract}
A vortex solid with self-generated randomness is found theoretically in a frustrated Josephson junction array (JJA) under external magnetic field with anisotropic couplings. Vorticies induced by external magnetic field develop stripes parallel to the direction of weaker coupling. It is shown analytically that there is a continuous, gapless band of metastable states in which stripes are deformed randomly by transverse undulation. The vortex solid with the frozen undulation in a metastable state freely slides along the direction of stronger coupling, thereby destroying ordering of phases even at zero temperature, but is jammed along the direction of weaker coupling.
\end{abstract}

PACS numbers: 61.43.Fs,62.20.Qp,74.81.Fa,74.25.Qt

Solids are systems with rigidity ranging from crystals, quasi-crystals to glasses and jammed granular matters 1]. Generally it becomes more challenging to understand mechanism of the formations of less periodic solids distinguishing them from liquids [2]. A useful guiding concept to study non-crystalline solids is frustration which inhibits simple crystallization. Imagine that there is a trick to "inject" dislocations artificially into a crystal from outside and that their density can be controlled at will. Such a system will provide a very interesting ground to study consequences of geometrical frustration, especially realization of self-generated randomness or glassiness without quenched disorder 3, 4]. Quite remarkably the Josephson junction array (JJA) under external magnetic field realizes such an ideal situation [5 [9]. Furthermore, transport properties of the JJA under external current can be regarded as "rheology" under external shear [10, 11].

JJA is a regular network of superconducting islands connected with each other by Josephson junction in the form, say, of a square lattice of size $N=L \times L$ as shown in Fig. 1 a) [5, 6]. The phases $\theta_{i}$ of the superconducting order parameter on the islands $i=1,2, \ldots, N$ interact with each other via Josephson coupling. Under magnetic field $B$, which can be varied at will in experiments, the number density $f=B a^{2} / \phi_{0}$ of vorticies (dislocations) can be forced into the configuration of the phases. Here $a^{2}$ is the area of a plaquette and $\phi_{0}$ is the flux quantum.

An interesting connection of JJA to the problem of frictions provides valuable insights. The frustrated JJA becomes essentially equivalent to the Frenkel-Kontorova (FK) model [12] and the two-chain model of Matsukawa and Fukuyama (MF) [13] in one dimensional limit $(N=$ $L \times 2)$, i. e. on the ladder lattice 11] [14]. These onedimensional systems are known to undergo a kind of jamming or frictional transition at zero temperature $T=0$, known as the Aubry's transition [15, 16] at a critical value of the strength of coupling $\lambda$ between two surfaces which

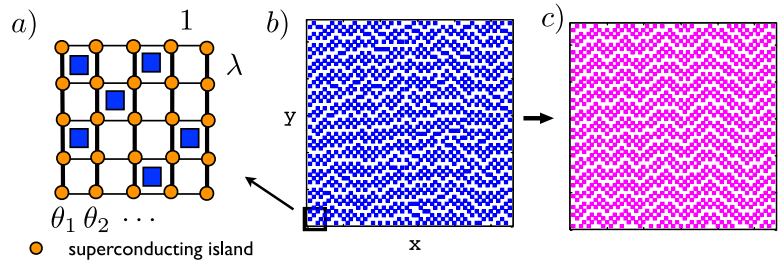

FIG. 1: Vortex patterns in an irrationally frustrated JJA under external magnetic field with anisotropic coupling. Here $\lambda=1.5$ so that the coupling is stronger along $y$ direction. Such an anisotropic JJA can be fabricated in laboratory by lithography technique(s) [9]. Panel a) displays the JJA on a square lattice. A fraction $f=21 / 55$, which approximates an irrational number $(3-\sqrt{5}) / 2=0.381966$.., of the plaquettes are occupied by vorticies with charge $1-f$ represented by filled squares. Panel b) displays an equilibrium vortex pattern at $T=0.2$ and Panel c) displays that at a nearby energy minimum reached via an energy descent algorithm.

are incommensurate with respect to each other. Then one would naturally be led to consider irrationally frustrated anisotropic JJA [10] with 1) irrational vortex density $f$ [7, 8] and 2) anisotropic couplings into $x$ and $y$ directions, say 1 into $x$ direction and $\lambda$ into $y$ direction.

In this Letter we study the ground state as well as low-lying states of the irrationally frustrated JJA with sufficiently strong anisotropy $\lambda \gg 1$. Numerically we found vortex stripes parallel to the direction of weaker coupling. In addition to the ground state in which vortex stripes are straight, we found numerous metastable states with different realizations of transverse undulation of the stripes as shown in Fig. 1. By a perturbative analysis in series of $1 / \lambda$ starting from infinite anisotropy limit $\lambda=$ $\infty$, we are able to reproduce a family of such low-lying metastable states including the ground state analytically. The coexistence of sliding and jamming in the system [10] is proved from the analytically constructed ground 
state and the low-lying states. Because of the sliding, the phases remain disordered even at $T=0$ for irrational $f$, in sharp contrast to JJA with rational $f$ where not only vorticies (chiralities for $f=1 / 2$ ) but also phases exhibit (quasi-)long ranged order at $T>0$ [17].

Model To simplify notations we label the vertices (superconducting islands) as $i=1,2, \ldots, N$ whose position in the real space is given by $\left(n_{i}, m_{i}\right)$. The properties of the JJA under the transverse magnetic field are known to be described by an effective classical Hamiltonian [5],

$$
H=-\sum_{<i, j>\| x \text {-axis }} \cos \left(\psi_{i j}\right)-\lambda \sum_{<i, j>\| y \text {-axis }} \cos \left(\psi_{i j}\right)
$$

where $\langle i, j\rangle$ denotes nearest neighbor and $\psi_{i j}$ the gauge-invariant phase difference, $\psi_{i j} \equiv \theta_{i}-\theta_{j}-A_{i j}$. The temperature $T$ is defined in a unit with $k_{\mathrm{B}}=1$. For the anisotropy $\lambda$, we need to consider only $\lambda>1$ by symmetry. The vector potential $A_{i j}\left(=-A_{j i}\right)$ is defined such that directed sum of them around each plaquette is $2 \pi f$.

Vortex charge $v_{i}$ of the vortex at the plaquette associated with the $i$-th vertex is defined by taking directed sum of $\left(\psi_{i j} / 2 \pi-\left[\psi_{i j} / 2 \pi\right]_{n}\right)$ on the junctions around the plaquette. Here $[x]_{n}$ denotes the nearest integer of the real variable $x$. It takes values $\ldots,-1-f,-f, 1-f, \ldots$. We use periodic boundary conditions so that the total vortex charge is enforced to be zero (charge neutrality).

It has been proposed that superconducting glass may be realized if $f$ is irrational [8]. While JJAs with rational $f$ develop periodic vortex lattices [17, 18], such simple orderings may be avoided with irrational $f$. Indeed equilibrium relaxations were similar to the primary relaxations observed in typical fragile supercooled liquids 19. Such a system is called as irrationally frustrated JJA. 25]

Numerical methods In numerical simulations, we used a series of rational numbers $p / q=5 / 13,8 / 21$, $13 / 34,21 / 55,34 / 89,55 / 144,89 / 233$ for the filling $f$, which approximate an irrational number $f=(3-$ $\sqrt{5}) / 2=0.38196601 \ldots$ Square lattices of size $L \times L$ with periodic boundary conditions for both directions are used. We choose $L=q$ so that the ratio $f=p / q$ converges to the target irrational number in $L \rightarrow \infty$ limit.

To generate the equilibrium ensemble, we used exchange Monte Carlo (MC) simulations combined with the over-relaxation method [20] performed on systems with $L=13-89$ using $20-120$ temperatures in the temperature range $T=0.2-0.4$. We used $10^{5}-10^{6} \mathrm{MC}$ steps for the equilibration and observations.

Stacked undulating vortex stripes As shown in Fig 1 the vorticies develop undulated stripes parallel to the direction of weaker coupling at low temperatures. The formation of the stripes is reasonable because the repulsive interactions between vorticies are anisotropic. A remarkable feature is that the stripes are stacked quite regularly along the stronger coupling as shown in Fig 1 c) in the nearby energy minima obtained via a simple en-
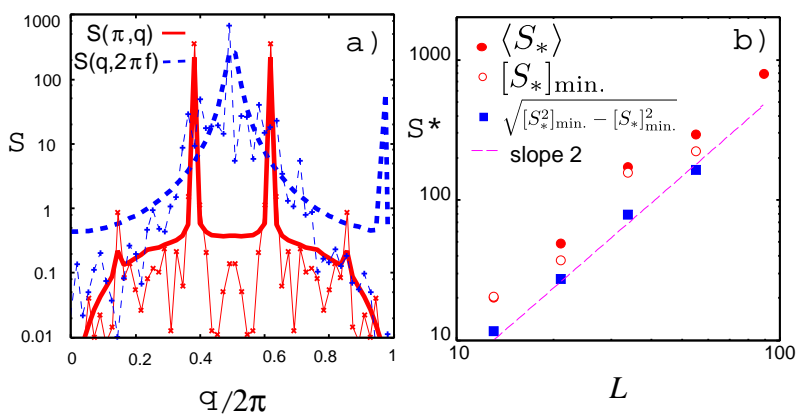

FIG. 2: Structure factor of vorticies. a) displays the crosssections of the structure factor $S\left(q_{x}, q_{y}\right)(L=55)$ with thermal average (thick lines) and at the energy minimum shown in Fig. 1 c) (thin lines). b) displays the amplitude of the peak of the structure factor $S_{*}=S(\pi, 2 \pi f)$ with thermal average $\left\langle S_{*}\right\rangle$, average over the energy minima $\left[S_{*}\right]_{\text {min. }}$ and variance of the minima-to-minima fluctuation $\sqrt{\left[S_{*}^{2}\right]_{\min }-\left[S_{*}\right]_{\text {min. }}^{2}}$. Here the average over minima $[. . .]_{\min }$ is taken over 100 energyminima obtained by independent initial conditions.

ergy descent algorithm. Starting from different thermalized configurations we obtained numerous energy minima similar to the one shown in Fig 1 c) but with different realizations of the transverse undulation. The nearly perfect stacking of the stripes strongly suggests that the energy barrier in going from one to another realization of significantly different undulation of vorticies (dislocations), which necessarily involve large number of plastic deformations, diverges with the system size so that the ergordicity is broken. This feature is markedly different from usual undulations found, for example, in liquid crystals which are fluctuating dynamically [21].

The stacked undulation is manifested in the structure factor of the vorticies. As shown in Fig. 2, the structure factor $S\left(q_{x}, q_{y}\right)$ exhibits prominent peaks at $\left(q_{x}, q_{y}\right)=(\pi, 2 \pi f)$ and $(\pi, 2 \pi(1-f))$ whose height scales with the system size as $N=L^{2}$ as usual Bragg peaks do. However the profile of the peak is peculiar: it decays sharply along $q_{y}$ reflecting the stacking but decays slowly by a power law $\left|\pi-q_{x}\right|^{-2}$ along $q_{x}$ reflecting the transverse undulation. Here we emphasize again that the transverse undulation is frozen in time. The frozen-in randomness is manifested in the minimum-to-minimum fluctuations of the structure factor shown in Fig 2 a) and b). Note that the variance of the fluctuation as well as the average grows linearly with the system size $N$ meaning that the structure factor is not self-averaging.

Analytic construction of the ground state Let us now turn to explicit construction of low-lying states by an analytical approach. To this end we propose a nontrivial ansatz for the ground state using the notion of the so called hull functions developed in the studies of the FK and MF models 12 16]. We propose that the gauge invariant phase differences $\psi_{i j}$ across the Josephson junctions, in the low-lying states of the anisotropic 
JJA $(\lambda>1)$ can be represented as,

$$
\psi_{(x, y)(x+1, y)}=\phi_{x}[y+\alpha(x)] \quad \psi_{(x, y)(x, y+1)}=\phi_{y}[y+\alpha(x)]
$$

Here $\phi_{x}[y]$ and $\phi_{y}[y]$ are functions defined on the "folded coordinate" $[y]=f y-\operatorname{int}(f y)$ where $\operatorname{int}(\mathrm{x})$ is the floor function. The folded coordinate takes values limited in the range $0 \leq[y]<1$. Such a function is called as a hull function 12, 15]. Note that if $f$ is irrational, which we always assume in this work, the vertices of the JJA uniformly fill the entire range of the folded coordinate $[y]$ in the limit $N \rightarrow \infty$. Thus we can treat $[y]$ as a continuous variable. Moreover one can then easily extract distribution of the values of the phase differences from the hull function because of the uniform distribution of $[y]$ over the support $0 \leq[y]<1$.

An obvious constraint is that the directed sum over $\psi_{i j}$ around each plaquette must be $-2 \pi f$. In addition, the Josephson currents must be conserved at each vertex (force balance condition) in each energy minimum. Thus the following two conditions should hold,

$$
\begin{aligned}
& \phi_{x}[y]+\phi_{y}[y+\delta(x)]-\phi_{x}[y+1]-\phi_{y}[y]=-2 \pi f \\
& \frac{1}{\lambda} \sin \phi_{x}[y]+\sin \phi_{y}[y]=\frac{1}{\lambda} \sin \phi_{x}[y-\delta(x)]+\sin \phi_{y}[y-1]
\end{aligned}
$$

where $\delta(x) \equiv \alpha(x)-\alpha(x-1)$.

Now our task is to look for the hull functions $\phi_{x}[y]$, $\phi_{y}[y]$ and phase differences $\delta(x)$ which satisfy the conditions on the plaquettes Eq. (3) and vertexes Eq. (4). We solve this problem by performing a $1 / \lambda$ expansion [16, 22 around the infinite anisotropy limit $\lambda=\infty$.

In the infinite anisotropy limit $\lambda \rightarrow \infty$ the weaker couplings can be neglected so that we easily find $\phi_{x}[y]=$ $(2[y]-1) \pi+O(1 / \lambda)$ and $\phi_{y}[y]=O(1 / \lambda)$ which trivially satisfies Eq. (3) and Eq. (4) (with $\lambda=\infty$ ). As such, the phase difference $\delta(x)$ is not fixed at this stage.

Let us consider first the ground state assuming that phase difference is uniform, i. e. $\delta(x)=\delta$. Using the above results in Eq. (44) we find $1 / \lambda$ correction term of $\phi_{y}[y]$, which in turn allows us to find $1 / \lambda$ correction term of $\phi_{x}[y]$ through Eq. (3). In this way we obtained analytic form of the hull functions up to $O\left(\lambda^{-3}\right)$ as, $\phi_{x}[y]=(2 f y-1) \pi+\frac{\left|a_{1}(\delta)\right|^{2}}{\lambda} s_{x}(1, y)+$ $\frac{\left|a_{1}(\delta)\right|^{4}}{8 \lambda^{3}}\left(\left|a_{3}(\delta)\right|^{2} s_{x}(3, y)-3\left|a_{1}(\delta)\right|^{2} s_{x}(1, y)\right)$ and $\phi_{y}[y]=$ $\frac{\left|a_{1}(\delta)\right|}{\lambda} s_{y}(1, y)+\frac{\left|a_{1}(\delta)\right|^{4}}{8 \lambda^{3}}\left(\left|a_{3}(\delta)\right| s_{y}(3, y)-3\left|a_{1}(\delta)\right| s_{y}(1, y)\right)$ with $a_{n}(\delta) \equiv\left(1-e^{-i 2 \pi f \delta}\right) /\left(1-e^{-i 2 n \pi f}\right), s_{x}(n, y) \equiv$ $\sin (2 n \pi f y)$ and $s_{y}(n, y) \equiv \sin \left(2 n \pi f y+\operatorname{Arg} a_{n}(\delta)\right)$. For irrational $f$, we find the energy is minimized by choosing $\delta=\delta^{*} \equiv 1 /(2 f)$ and obtain the ground state energy $E_{\mathrm{g}}$, $\frac{E_{\mathrm{g}} / \lambda}{N}=-1-\frac{\left|a_{1}\left(\delta^{*}\right)\right|^{2}}{4 \lambda^{2}}-\frac{\left|a_{1}\left(\delta^{*}\right)\right|^{4}}{16 \lambda^{4}}\left(\frac{1}{4}-\left|a_{1}\left(\delta^{*}\right)\right|^{2}\right)+O\left(\lambda^{-6}\right)$. The vortex configuration of the ground state is indeed the stripes like Fig. 1 1 but without the transverse undulation.

Band of undulated metastable states Next let us construct the low-lying states with transverse undulation of stripes shown in Fig. 1 Somewhat

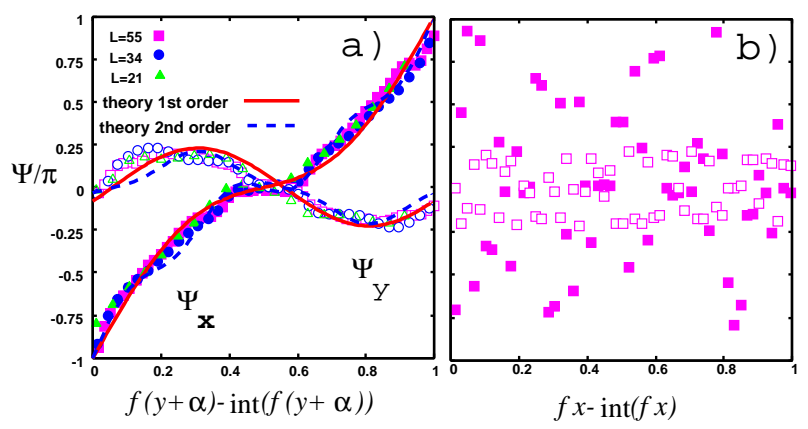

FIG. 3: Configuration of the gauge invariant phase differences across Josephson junctions in energy minima. Here the anisotropy is $\lambda=1.5$. In panel a) original $y$ axis is "folded" to $[y]=f y-\operatorname{int}(f y)$. The symbols are data of the gauge invariant phase differences $\psi_{x}=\psi_{(x, y)(x+1, y)}$ (filled symbols) and $\psi_{y}=\psi_{(x, y)(x, y+1)}$ (open symbols) across Josephson junctions parallel to $x$ and $y$ axis. Each data set consists of data points on a 'column' $(x, 1),(x, 2), \ldots,(x, L)$ at an arbitrary chosen $x(1 \leq x \leq L)$ in an arbitrary chosen energy minima respectively. Each data set is shifted globally by some $\alpha$ so that different data sets collapse on top of each other. The lines are analytically obtained hull functions of the ground state. In panel b) the data on the same energy minimum $(L=55)$ are plotted against "folded" $x$-axis.

surprisingly, we can solve Eq. (3) and Eq. (4) with arbitrary $\delta(x)$, finding, $\phi_{x}[y]=(2 f y-1) \pi+$ $\left.-\frac{\left|a_{1}\left(\delta^{*}\right)\right|^{2}}{4 \lambda}\left[C_{1}[\delta(x)] \cos (2 \pi f y)+C_{2}[\delta(x)] s_{x}(1, y)\right)\right] \quad+$ $O\left(\lambda^{-2}\right)$ and $\phi_{y}[y]=\frac{\left|a_{1}\left(\delta^{*}\right)\right|}{2 \lambda}\left(s_{y}(1, y-\delta(x)+1 /(2 f))+s_{y}(1, y)\right)+$ $O\left(\lambda^{-2}\right)$ with $C_{1}[\delta(x)] \equiv-\sin (2 \pi f \delta(x+1))-\sin (2 \pi f \delta(x))$ and $C_{2}[\delta(x)] \equiv-2+\cos (2 \pi f \delta(x+1))+\cos (2 \pi f \delta(x))$.

As the result the energy becomes, for irrational $f, \frac{E_{\mathrm{g}}}{\lambda}+$ $L \frac{\left|a_{1}\left(\delta^{*}\right)\right|^{2}}{8 \lambda^{2}} \sum_{x=1}^{L}(1+\cos (2 \pi f \delta(x)))+O\left(\lambda^{-4}\right)$. It is evident that there exists a gapless, continuous spectrum of lowlying states each of which is parametrized by a function $\delta(x)$. Assuming $|\delta(x)-1 /(2 f)| \ll 1$, we obtain an one dimensional elastic Hamiltonian with an unusual elastic constant which grows linearly with the system size $L$.

Let us emphasize that the undulated states with the arbitrary $\delta(x)$ are ensured to satisfy the force balance condition Eq. (41). Thus the system trapped in an undulated state cannot relax spontaneously down to the ground state since they are metastable: the undulation is distinct from "phonons" by which vorticies cannot move.

Now the unusual structure factor of the vorticies $S\left(q_{x}, q_{y}\right)$ in Fig. 2 can be understood as follows. The configuration of the ground state is a function of the folded coordinate $[y]$ so that $S\left(q_{x}, q_{y}\right)$ must have peaks along the $q_{y}$-axis at $q_{y}=2 \pi f$ and $2 \pi(1-f)$. On the other hand the phase shift of the hull function $\delta^{*}=1 /(2 f)$ along $x$-direction meaning that $S\left(q_{x}, q_{y}\right)$ must have a single peak along the $q_{x}$ axis at $q_{x}=\pi$, reflecting the horizontal stripes. The power law tail $\left|q_{x}-\pi\right|^{-2}$ naturally follows from the effective one-dimensional elastic Hamiltonian for the transverse undulation obtained above. 
In Fig. 3 we plot the phase differences across the junctions in energy minima obtained numerically and compare them with the hull functions obtained analytically. For simplicity we show here the hull function for the ground state disregarding small differences due to the undulation. Our perturbative result grasps well the overall features. The agreement will be further improved by taking into account higher order terms in the $1 / \lambda$ expansion.

A remarkable consequence of the analytic hull function is that the undulated vortex solid can freely slide into the $y$ direction: Given an energy minimum described by the hull functions $\phi_{x}[y]$ and $\phi_{y}[y]$, a family of solutions with exactly the same energy can be obtained through the operation $[y] \rightarrow[y+\alpha]$ with varying phase shift parameter $\alpha$. Consequently the phases must remain disordered even at $T=0$. As shown in Fig. 3 a) the phase differences across junctions parallel to the $x$-axis take all possible values in the range $-\pi \leq \phi_{x}[y]<\pi$ meaning that the system can be sheared indefinitely along the $x$-axis which amount to unidirectional sliding of the undulated vortex solid into the $y$-direction with fixed pattern. In contrast, the plot against "folded" $x$-axis shown in Fig. 3 b) exhibits no hint of a single valued, regular hull function. Also note that the distribution of the $\phi_{y}$ does not span the entire range $-\pi \leq \phi_{y}<\pi$ which is needed to allow shear along $y$-axis measning jamming along $x$-axis.

It is instructive to compare the above results with the FK model. In the FK model the hull function is proved to be an analytic function in the sliding phase $\lambda<\lambda_{c}$ but becomes non-analytic in the jammed phase $\lambda>\lambda_{c}$ [15]. In the anisotropic JJA, the sliding and jamming are dual in the sense that they are simultaneously taking place along different axes. Indeed in [10] it was found numerically that the shear-modulus is zero/finite along the direction of weaker/stronger coupling at $T=0$. Furthermore it was suggested that the symmetric point $\lambda=1$ is a critical point at zero temperature $\lambda_{c}(T=0)=1$ similar to the jamming point in granular matters [1]. Indeed recent studies at finite temperatures suggest $T_{c}(\lambda=1)=0$ [23, 24]. On the other hand the growth of the peak height of the vortex structure factor with the system size $L$ (Fig. 2) suggests $T_{c}(\lambda)>0$ at least at $\lambda \gg 1$.

The analogy to the FK model suggests that the analytic hull functions $\phi_{x(y)}[y]$ becomes non-analytic at the critical point $\lambda=\lambda_{c}=1$ and remains non-analytic for $\lambda<1$. (The other way around for $\phi_{x(y)}[x]$ ). However we speculate that the $1 / \lambda$ expansion, which yields only analytic hull functions, remains stable up to $\lambda=1$. Then certain "non-perturbative" solution(s) of Eq. (3) and Eq. (4) must emerge at weaker anisotropy and make level crossing(s) with the horizontal stripe state.

To conclude we found undulated vortex stripes in irrationally frustrated Josephson junction array with anisotropic Josephson coupling theoretically. It will be very interesting to study critical properties of the system closer to the symmetric point where the present pertur- bative approach should break down.

Acknowledgement We thank Hikaru Kawamura, Jorge Kurchan and Hiroshi Matsukawa for useful discussions. We thank the Supercomputer Center, ISSP, University of Tokyo for the use of the facilities. This work is supported by Grant-in-Aid for Scientific Research on Priority Areas "Novel States of Matter Induced by Frustration" (1905200*) and Grant-in-Aid for Scientific Research (C) (21540386).

[1] C. S. O'Hern et. al., Phys. Rev. E 68, 011306 (2003).

[2] J. Kurchan and D. Levine, arXiv:0904.4850 1.

[3] Geometrical frustration, J. F. Sadoc, Rémy Mosseri, Cambridge University Press, 1999.

[4] G. Tarjus, S. A. Kivelson, Z. Nussinov and P. Viot, J. Phys. Condens. Matters 17 R 1143 (2005).

[5] Introdunction to Superconductivity, M. Tinkham, Courier Dover Publications (2004).

[6] H. S. J. van der Zant et. al., J. Low. Temp. Phys., 8267 (1991); R. S. Newrock et. al., Solid State Phys. 54, 263 (2000).

[7] S. Teitel and C. Jayaprakash, Phys. Rev. Lett. 51, 1999 (1983).

[8] T. C. Halsey, Phys. Rev. Lett. 55, 1018 (1985).

[9] S. Saito and T. Osada, Physica B: Condensed Matter Vol 284-288, 614 (2000).

[10] H. Yoshino, T. Nogawa, and B. Kim, New J. Phys. 11, 013010 (2009).

[11] H. Yoshino, T. Nogawa, and B. Kim, Prog. Theor. Phys. Supple. 184153 (2010).

[12] L. M. Floria and J. J. Mazo, Advances in Physics 45, 505598 (1996) and "The Frenkel-Kontorova Model - Concepts, Methods and Applications", O. M. Braun and Y. S. Kivshar, Springer (2004).

[13] H. Matsukawa and H. Fukuyama, Phys. Rev B. 49, 17286 (1994).

[14] J. J. Mazo et. al, Phys. Rev. B 52, 10433 (1995); C. Denniston and C. Tang, Phys. Rev. Lett. 75, 3930 (1995).

[15] M. Peyard and S. Aubry, J. Phys. C, 16, 1593 (1983).

[16] T. Kawaguchi and H. Matsukawa, Phys. Rev. B 56, 13932 (1997).

[17] S. Teitel and C. Jayaprakash, Phys. Rev. B 27, 598 (1983).

[18] T. C. Halsey, Phys. Rev. B 31, 5728 (1985); P. Gupta et. al., Phys. Rev. Lett. 80, 105 (1998); C. Denniston and C. Tang, Phys. Rev. B 60, 3163 (1999).

[19] B. Kim and S. J. Lee, Phys. Rev. Lett. 78, 3709 (1997).

[20] K. Hukushima and K. Nemoto, J. Phys. Soc. Jpn 65, 1604 (1996), M. Creutz, Phys. Rev. D 36, 515 (1987).

[21] Principles of condensed matter physics, P. M. Chaikin and T. C. Lubensky, Cambridge University Press (1995).

[22] T. Nogawa and K. Nemoto, Phys. Rev. B 73, 184504 (2006).

[23] S. Y. Park et. al, Phys. Rev. Lett. 85, 3484 (2000).

[24] E. Granato, Phys. Rev. Lett. 101, 027004 (2008).

[25] A system with e. g. $f=1 / 2+\epsilon$ with infinitesimal irrational $\epsilon$ should behave as a system with $f=1 / 2$ in some sense. Although this is an interesting issue, we focus on properties of systems with irrational $f$ in $N \rightarrow \infty$ limit. 